\title{
Educación Superior en Emprendimiento y la Superación de la Pobreza ${ }^{1}$
}

\author{
Higher Education in Entrepreneurship \\ and Overcoming Poverty
}

Ricardo Pérez Sáenz²

\section{Resumen}

La educación en emprendimiento fomenta cultura emprendedora y la empresarialidad, por ello, en Colombia es obligatoria esta cátedra en los establecimientos educativos. Las universidades colombianas adoptan este lineamiento, brindando educación sobre emprendimiento, para emprender e innovar, para mantener y desarrollar las empresas. ¿Cómo lo hacen?, ¿qué tipo de emprendimientos fomentan?, ¿la cultura emprendedora y la empresarialidad que fomentan puede aportar a la superación de la pobreza, al crecimiento socioeconómico y al desarrollo humano?, son preguntas que permitieron la construcción de este artículo y el uso de un tipo de investigación descriptiva y explicativa, el análisis de universidades comprometidas con el emprendimiento, determinando, que enseñan emprendimiento económico, social, de base tecnológica y ambiental, promoviendo emprendimiento interno y externo, con lo cual, sus estudiantes y egresados han logrado aumentar la efectividad productiva y la creación de empresas, contribuyendo a la superación de la pobreza monetaria y al desarrollo humano de estos.

Palabras clave: Educación Superior; Educación en Emprendimiento; Cultura emprendedora; Desarrollo; Pobreza.

\begin{abstract}
Entrepreneurship education promotes entrepreneurial culture and entrepreneurship, and in Colombia this institution is compulsory in educational establishments. Colombian universities adopt this approach, providing education on entrepreneurship, to undertake and innovate, to maintain and develop companies. How do they do it? What kind of entrepreneurship do they foster? Can the entrepreneurial culture and entrepreneurship that they foster contribute to overcoming poverty, socioeconomic growth and human development? These are questions that allowed the construction of this article and the use of a type of descriptive and explanatory research, the analysis of universities committed
\end{abstract} to entrepreneurship, determined, that teaches economic, social, technological and environmental entrepreneurship, promoting internal and external entrepreneurship, with which students and graduates Have been able to increase the productive effectiveness and the creation of companies, contributing to the overcoming of the monetary poverty and to the human development of these.

Keywords: Higher Education; Entrepreneurship Education; Entrepreneurial culture; Development. Poverty.

' Artículo resultado de investigación, fruto de la construcción del estado del arte, en el marco del proyecto de tesis doctoral: La educación superior y el desarrollo de cultura emprendedora en los estudiantes de las facultades de Ciencias Económicas, Administrativas y Contables de la Universidad de Cartagena y de la Universidad Tecnológica de Bolívar, 2006-2015 para el doctorado en Ciencias de la educación RUDECOLOMBIA de la Universidad de Cartagena, en la Línea de formación doctoral: Educación superior: currículo, gestión y evaluación. Bajo la doctora Rina De León Herrera.

${ }^{2}$ Economista, especialista en Gerencia de Recursos Humanos, MGO Magister en Gestión de Organizaciones - EAN, M.Sc. Magister en Ciencias - Gestión Organizacional, Universidad de Quebec - Canadá. E-mail: ricardo3011@hotmail.com 


\section{Contexto de la investigación}

La Real Academia de la Lengua, epistemológicamente define que la palabra educación viene del latín educere «sacar, extraer» o educare «formar, instruir», también se afirma que viene del latín educatio. - onis, el cual indica acción y efecto de educar. Etimológicamente implica una acción con un propósito, reconociendo su importancia e impacto en quien la recibe. Al respecto Pitágoras, indicó, que la educación es templar el alma para las dificultades de la vida, en este sentido propuso el proceso educativo como el escenario para desarrollar condiciones por medio de las cuales el hombre enfrente sus problemas e implemente soluciones.

La educación es un proceso humano y cultural, que implica formar e instruir, generándose con ello perfiles de egreso caracterizados por competencias investigativas, emprendedoras, innovadoras, socio humanistas e integrales, esto significa que la educación es un proceso que favorece el desarrollo intelectual, moral y afectivo de un individuo o grupo de personas, preparándolo para conocer mejor la realidad, entenderla y tomar decisiones que impliquen aprovechar las oportunidades y corregir las imperfecciones del funcionamiento del sistema del cual es parte fundamental.

En la Constitución Política de Colombia 1991(artículo 67, 1991:27) y la ley 30 de 1992, se establece "la Educación como un derecho de la persona y un servicio público, que tiene una función social", esto implica un gran compromiso con el hombre y la sociedad que este integra. En este orden de idea, la Ley (Ley 115de 1994) y (Ley 749 de 2002), define la Educación como un proceso de formación permanente, personal, cultural y social que se fundamenta en una concepción integral de la persona, humana, y en sus derechos.

El Artículo $1^{\circ}$ de la ley 30 de 1992 , establece que la Educación Superior es un proceso permanente que posibilita el desarrollo de las potencialidades del ser humano de una manera integral, por tanto, la educación que se imparta en las universidades debe contribuir a que el potencial de cada estudiante se convierta en una capacidad. En el Artículo $4^{\circ}$ responsabiliza a la educación, del despertar en los educandos un espíritu reflexivo, orientado al logro de la autonomía personal. En este sentido surge la preocupación por saber, si la educación superior que se imparte en Colombia, fomentan o no la autonomía y la libertad, que en términos de empleabilidad y empresarismo, significa la no condición de empleado, sino por el contrario, creador, dueño o líder de sus propia empresa (La Educación Superior en Colombia no tiene como finalidad facilitar la creación de empresas, pero al formar y trabajar por desarrollar cultura emprendedora en los estudiantes, se puede constituir, en medio para lograr que la creatividad, la invención y la innovación se materialicen en un aparato productivo, que genere empleo, cree valor y riqueza y contribuya así, al desarrollo humano.

En la ley 30 de 1992, Artículo $6^{\circ}$ se plantearon los objetivos de la Educación Superior y de sus instituciones. En este contexto, aumenta el interés por indagar acerca de cómo las IES colombianas, vienen impartiendo educación que cumpla con estos propósitos de formar integralmente, desarrollar capacidades humanas, capital humano y competencias que les permitan a los educandos ayudar a la solución de problemas tales como la pobreza, aportando así al desarrollo socioeconómico de ellos (calidad de vida, desarrollo humano y bienestar) y de quienes habitan su entorno.

\section{La educación y la superación de la pobreza}

A finales de los años 90 del siglo XX, y en los inicios del siglo XXI, los indicadores de pobreza en el mundo parecieron deslegitimar el modelo de crecimiento y desarrollo económico mayoritaria- 
mente implementado, así mismo colocaron en entredicho las políticas y las estrategias hasta entonces utilizadas con la firme intención de contribuir a la lucha contra la pobreza extrema, el hambre, la exclusión y la desigualdad en los ámbitos rurales o urbanos de una nación.

A las autoridades gubernamentales en el mundo les rondaba más fuerte que nunca la pregunta: ¿tiene solución la pobreza?, En este orden de ideas, surge, en el reciente mundo globalizado, la necesidad de analizar la relación educación - pobreza. Para tal fin, es necesario recordar que entre los años 40 - 80 la teoría de la modernización, la teoría de la dependencia y su modelo de crecimiento económico consideraron que la educación, elevaba el capital humano y como tal este aumentaba la productividad, adicionalmente entre más cultos y educados fueran los seres humanos, más probabilidades de participación en política se generarían, mejoraría la riqueza cultural de los individuos y como tal se podrían mejorar sus relaciones sociales. Por tanto, invertir en educación se consideraba importante desde la perspectiva social, económica, política y cultural, de tal manera que con mejores conocimientos, habilidades, destrezas y competencias en general, las personas podrían contribuir significativamente a la modernización del mundo, haciéndolo más equitativo, menos excluyente, pobre, vulnerable y más desarrollado.

En la década de los ochenta surge la teoría neoliberal. Esta planteó que una nación solo podría conseguir un crecimiento económico sostenible, realizando cambios estructurales, que incluyeran la implementación de políticas públicas tales como las privatizaciones, medidas de descentralización, la reducción del gasto público y la liberalización financiera y comercial. Se puede afirmar que el consenso de Washington (Se entiende por Consenso de Washington un listado de políticas económicas consideradas durante los años 90 por los organismos financieros internacionales y centros económicos, con sede en Washington D.C), se constituyó en el mayor referente de las políticas neoliberales, que posteriormente fueron lideradas por el Banco Mundial. Las privatizaciones incluyeron las instituciones de educación media y superior, partiendo del supuesto, de que la educación privada era de mayor calidad que la pública, adicionalmente los recortes en el gasto público, significaron menos recursos para las políticas y estrategias de ampliación de cobertura e inversión en educación. Así mismo, el paradigma económico se modificó, por tanto, se pasó de pensar que los problemas de crecimiento y de desarrollo que experimenta un país, era exclusivamente nacional, por el contrario, problemas como la pobreza, pasaron a convertirse en temas que debían ser parte de una agenda internacional y como tal, las estrategias de mitigación deberían ser conjuntas, de consenso y además de implementación global.

En este sentido una familia debía decidir si los miembros realizarían estudios superiores o por el contrario trabajarían para ayudar con recursos a la financiación de la canasta familiar, en virtud de los millones de empleos que se perderían producto de las privatizaciones o los recortes en el gasto público, es decir, se realizaba una especie de función costo - beneficio, evaluando con ello los costos de estudiar, versus los beneficios que el futuro recibirían, mejorando sus posibilidades de empleabilidad. Es en este contexto el emprendimiento y en especial la educación emprendedora aparece y ganan mucha credibilidad.

La educación tiene como finalidad desarrollar la capacidad intelectual, moral y afectiva, formar e instruir, desarrollar las capacidades cognitivas y físicas de las personas, de tal forma que estos puedan integrarse plenamente a la sociedad, convivir y convertirse en un verdadero aprendizaje para la vida, facilitando la inclusión, la mitigación de la marginación y aportando de manera contundente al desarrollo humano y superación de la pobreza. 
En este contexto, iniciamos confirmando la relación natural que existe entre la educación y la pobreza, lo cual se puede explicar desde diferentes perspectivas, siendo el camino más adecuado, hacer inicialmente referencia a las funciones sociales que tiene la educación.

La educación desde la perspectiva de sus funciones sociales, nos permite abordar la conexión existente entre esta y el desarrollo. Con estos elementos nos acercarnos a la comprensión de cómo la educación puede ser útil de cara a corregir la existencia de diferentes problemas que aquejan a la humanidad y que no permiten su desarrollo (Avellaneda, 2007). Uno de esos problemas es la pobreza, la cual ha intentado ser superada, desde la implementación de diferentes modelos, teorías, enfoques y paradigmas, entre los principales se encuentra la teoría del crecimiento económico, la teoría del modernismo, la teoría del desarrollo humano, la teoría del capital humano, la teoría de las capacidades y otras de donde se identificaron, diseñaron, seleccionaron, e implementaron estrategias para mitigar la pobreza. Sin embargo es desde la década de los 90 del siglo pasado, cuando se genera un consenso mundial en considerar la inversión en educación, la principal vía para la superación de la pobreza. (Tarabini, 2008), (Larrañaga, 1997), (Sen A. K., 1976), (Bonal X. y., 2006), (Bonal, Tarabini, \& Klickowsky, ¿Puede la educación erradicar la pobreza? , 2006), (Bonal, X, 2004).

Es necesario que las universidades reconozcan que la investigación, el emprendimiento y la innovación, deben estar focalizadas y al servicio de la identificación del conocimiento, la socialización, la apropiación, la implementación y evolución del mismo, creando un nuevo conocimiento y suficiente información, que con un responsabilidad social universitaria, facilite el surgimiento de nuevas organizaciones sociales y económicas, más equitativas e incluyentes (Arendt, Cruz, \& Novales, 1993). En este sentido, la educación superior debe preparar hoy al hombre que un futuro este en capacidad para investigar su realidad, emprender sobre ella e innovar, de tal manera que la transforme, a través de bienes y servicios y lo debe hacer con mucha sensibilidad, tacto y compromiso social, para conducir al sistema al estado equitativo $y$ con justicia social que se requiere. Por tanto la universidad es fundamental para la transformación de la humanidad y en especial de la sociedad que este integra, al respecto su misión debe estar cimentada en el principio de "educar en la ciencia" (Boni Aristizábal, 2000), fortaleciendo el capital Humano (Becker, 1993), siendo un instrumento que convierte las potencialidades en verdaderas capacidades, las cuales deberán orientarse a la generación de riqueza social, económica y tecnológica, donde el objetivo sea la felicidad del educado. Por otro lado, existen autores que sostienen que las universidades deben suministrar educación liberal (Nussbaum, 1997), (Habermas, 2000) cuyo propósito sea la mejora de la calidad de vida.

Las universidades son determinantes en la generación, creación, divulgación, uso y aplicación del conocimiento existe y el nuevo (Salmi, 2003) (Banco Mundial, 2003), por intermedio de la educación terciaria o universitaria, se logra el desarrollo y el fortalecimiento de competencias técnicas u operativas, tácticas, humanas, de gestión, conceptuales y estratégicas, de carácter profesional, a través de las cuales se puede mejorar la efectividad productiva de una organización, la creación de nuevas y la productividad, competitividad y sostenibilidad de aparatos productivos, por intermedio de ventajas competitivas, que redundan en generación de empleos de calidad, de riqueza monetaria y capacidad para satisfacer necesidades básicas que permitan superar pobreza y otros problemas que enfrenta la humanidad (Walker, 2006; Nussbaum, 1997). Al respecto de esta relación entre la educación superior, el papel de quien la dinamiza, en este caso las universidades (IES) y la 
pobreza, también encontramos los aportes de Coit Gilman, la tarea de la universidad debe ser: Reducir la miseria de los pobres, la ignorancia en la escuela, el fanatismo en el templo, el sufrimiento en el hospital, el fraude en los negocios y la locura en la política (Harkavy, 2006, p. 10).

\section{La educación superior en emprendimiento, la cultura emprendedora y la empresarialidad}

S. Covey (1999) según Gutiérrez Montoya, 2011, realiza aportes importantes, en cuanto a la educación en emprendimiento, la cultura emprendedora y la empresarialidad que se despliega, teniendo la capacidad y competencias emprendedoras, al respecto plantea que "la verdadera máquina que está conduciendo nuestra economía y sociedad son los emprendedores". Así mismo, el estudio Global Entrepreneurship Monitor (GEM) (2010) ha demostrado la importancia que el tema del espíritu empresarial ha adquirido, y se ha llegado a convertir en una herramienta para la creación de empleo y generación de riqueza, lo cual demuestra que el crecimiento y el desarrollo económico están ligados al emprendimiento (Acs, Arenius \& Minniti, 2005; José C. Sánchez, 2011).

Los antecedentes legales del emprendimiento en Colombia se señalan en la ley 29 de 1990, llamada Ley de la Ciencia y la Tecnología, la cual se constituye en el marco jurídico que regula las disposiciones para el fomento de la investigación científica y el desarrollo tecnológico. Se puede señalar como la ley que da soporte a las incubadoras de empresas y parques tecnológicos en Colombia. Para el caso especial de incentivar el desarrollo de la innovación y el emprendimiento, la ley 29 del 90 y los Decretos 393 y Decreto 585 del 91, reglamentan los proyectos de innovación que incorporan tecnología, creación, generación, apropiación y adaptación de la misma. Sin embargo, los mayores avances legislativos en la materia llegan en el siglo XXI se dan con la ley 590 del 10 de julio de 2000, conocida como ley MIPYME, y posteriormente con la ley de fomento a la cultura emprendedora, ley 1014 de 2006.

La ley 1014 de 2006 en Colombia, también denominada la ley del emprendimiento, definió la cultura como un conjunto de valores, creencias, ideologías, hábitos, costumbres y normas que comparten los individuos y que se reflejan en los patrones de comportamiento de la sociedad y las organizaciones que la integran. Así mismo contempla la formación en emprendimiento como la vía para desarrollar la cultura emprendedora, planes de negocios y en general lo que en la ley se denomina "empresarialidad", es decir el despliegue de la capacidad creativa de las personas sobre la realidad que lo rodea.

Existen personas, pioneras, aventureras, flexibles, dinámicas, capaces, creativas e innovadoras, a quienes se suele reconocer como emprendedores, este concepto históricamente ha sido relacionado con el vocablo francés entrepreneur, introducido por el economista Cantillon (nacido probablemente el año 1680 en Irlanda y fallecido en 1734, La obra maestra de este autor, es sobre economía, fue ampliamente acreditado por ser el primero en describir el proceso de mercado impulsado por el espíritu empresarial, lo cual le significa para muchos científicos y expertos, el ser merecedor y llamarlo "el Padre de la economía de empresa"). El economista (Say, 1803) planteo que un emprendedor es un agente económico que une todos los medios de producción y produce un producto, que, mediante la venta, recupera los costos y obtiene un excedente. (Schumpeter, 1934): índico que los emprendedores son innovadores que buscan destruir el estatus-quo de los productos y servicios existentes para crear nuevos productos y servicios, por tanto, un emprendedor busca el cambio, responde a él y explota sus oportunidades. 
Los emprendedores promueven la innovación y la creatividad (Marinelli, 1998; Casson, 1982). McGinnis (1987) establece que un emprendedor "cree en la innovación y es creativo, pero con una imaginación pragmática”.

En este contexto, el concepto de emprendimiento evoluciona y se le relaciona con la innovación, entendida esta como una herramienta específica de un emprendedor para convertir una fuente en un recurso (Drucker, 1964 ). El emprendimiento es utilizado por una persona para hacer que la innovación, la flexibilidad y la creatividad sean operacionales (Castillo, 1999, p. 4; Shapero, 1982). En este sentido, emprender es perseguir la oportunidad más allá de los recursos que se controlen en la actualidad (Stevenson H, 2000), para lograrlo es necesario ver lo que todos ven, pensar lo que algunos piensan y hacer lo que nadie hace (Primer Congreso Provincial sobre Emprendedorismo y Desarrollo Local, Pigüe, 2003), a partir de estas acepciones Schumpeter describió al emprendedor como una persona extraordinaria que promovía nuevas combinaciones o innovaciones, no es el que inventa, el que consigue que las cosas se Hagan. (Shumpeter, 1950). Por tanto el emprendedor debe poseer espíritu empresarial (Varela \& Bedoya, 2006), entendido como la capacidad para pensar, razonar y actuar centrado en las oportunidades, planteada con visión global y llevada a cabo mediante un riesgo calculado.

Partiendo de la base, de que la cultura emprendedora sea educable, surgieron diferentes planteamientos acerca de los tipos de emprendimiento que se deberán promover y enseñar, las actitudes que son necesarias desarrollar en el individuo y futuro emprendedor. Por esta razón, las escuelas se dedicaron, y aun lo hacen, a enseñar de emprendimiento y otras a desarrollar las capacidades necesarias para lograrlo. La escuela de Schumpeter establece como retos de la educación en emprendimiento, el desarrollo de la capacidad para investigar, ser creativo e inventivo, facilitando la evolución de la principal competencia de un emprendedor, es decir, la innovación. Por su parte en la escuela Austriaca (las principales contribuciones Ludwig Von Mises y de Israel Kirzner), se sostienen que los cambios generados por la actividad emprendedora tienden a equilibrar los mercados. Por tanto, se debe enseñar más un emprendimiento de tipo social. De la universidad de Harvard, Howard Stevenson, interpretó la mentalidad emprendedora como un sistema de gestión apoyado más en las oportunidades que en los recursos. En la Escuela de Chicago Los mayores aportes fueron realizados por el economista Frank Knight, en su teoría de del empresariado plasmada en el (Knight F. , 2006) (correspondiente a su tesis doctoral), en ella sostiene que la educación para formar a un emprendedor debe desarrollar en él, la capacidad de adaptación en situación de incertidumbre, capacidad para optimizar recursos y generación riqueza. Por otro lado, encontramos la escuela dinamizada por Timmons, quien construye un modelo educativo que se basa en las capacidades adquiridas y no en la selección a priori de personas con la antiguamente llamada capacidad emprendedora. Ha defendido que los emprendedores no nacen, sino que se forman, sin embargo, manifiesta que preferiblemente en los ámbitos de la educación superior, resulta más fácil desarrollar competencias para emprender. (Timmons, 1994).en conclusión, los planteamientos de las diferentes escuelas permiten establecer que La enseñanza del emprendimiento no está ligada al número de empresas creadas, o a las pequeñas y medianas empresas, está ligada a (Castillo, 1999): La facultad de crecer y de crear riqueza (schumpeter - desequilibrios), La orientación al crecimiento (teoría de Drucker y Stevenson) y al encaje de tres puntos básicos: las personas, el mercado y los recursos. Tomando en cuenta este recorrido por los aportes de las diferentes escuelas que han surgido alrededor de la 
promoción, enseñanza e implementación del emprendimiento, a continuación, abordamos el tema desde lo acontecido en Colombia.

\section{El emprendimiento, el crecimiento económico y la superación de la pobreza}

Existen aportes teóricos muy valiosos en la literatura científica divulgada en el siglo pasado y en el presente en lo referente al papel del emprendimiento en el crecimiento y desarrollo económico de las ciudades, regiones o un país, se destacan los siguientes autores: (Cantillon, 1755), (Stuart Mil, 1803), (Baptistsay, Schumpeter, \& Baumol, 1993), (Kirzner I. M., 1973), (Knight F. , 1921), y más recientemente los trabajos de (Audretsch, 2009). Estos autores desarrollaron la noción del capital emprendedor en la sociedad y sostienen que se trata de otro factor productivo que contribuyen al crecimiento y desarrollo económico, en forma análoga al capital físico y humano, sustentando que la innovación que promueve un emprendedor, es determinante en el proceso de conversión del conocimiento tecnológico, fruto de la investigación, que es capaz de generar nuevos productos, servicios, puestos de trabajo estimulando así él crecimiento y desarrollo económico.

Es en este marco de referencia normativa y conceptual, que resulta necesario, traer a colación diferentes teorías, tales como la teoría del desarrollo humano y de las capacidades de Sen (Sen A. , 2000) (El desarrollo humano integral es "el proceso de expansión de las capacidades de las personas que amplían sus opciones y oportunidades" (PNUD, 2007). En este sentido, el desarrollo humano integral está relacionado con el fortalecimiento de capacidades desde todas las dimensiones del ser humano, que le permitan generar acciones en todos los ámbitos de su vida en pro de su progreso y bienestar), la teoría del capital humano (Becker, 2009) y la teoría del capital emprendedor (Audretsch, 2009).

El economista Becker, introdujo en el año 1964 la teoria del Capital Humano, esta produccion científica, que lo hizo ganador del Nobel en 1969, establece una "relacion entre educacion, el conocimiento, ciencia y tecnologia, lo cual indicaba que el motor de desarrollo de un pais son las ideas y la aplicacion de la tecnologia sobre las habilidades, aprendizaje y capital humano, es decir, el conocimiento, habilidades y capacidades que posee, desarrolla y acumula cada persona en su trayectoria universitaria o de formacion, asi como la laboral y organizacional... el capital humano se entiente como la inversion en conocimientos, formacion e informacion, lo que permite obtener un mayor rendimiento y productividad" (Torres, 2009, p. 65-81).

Audretsch, plantea que la investigación genera nuevo conocimiento, para introducirlo a los mercados, a través de innovaciones, demanda de la invención y la creatividad, siendo esto último el capital emprendedor que teje el puente o el paradigma que articula la investigación con la innovación. Así mismo sugiere entender el capital emprendedor como un nuevo factor de producción, capaz de estimular la efectividad productiva a nivel interno, la creación de nuevas empresas, trabajos y producción a nivel externo y como tal estimular el crecimiento y el desarrollo económico (Audretsch, D. 2009, p. 33).

A partir de los referentes planteados resulta importante describir y explicar cómo la Universidad Escuela de Administración de Negocios EAN, la Universidad ICECI, EAFIC, el CESA, Los Andes, la Universidad del Norte, La Universidad Tecnológica de Bolívar, a nivel privado y la Universidad Nacional, la Tecnológica de Pereira, la del Valle, la de Antioquia, la Universidad de Cartagena, entre otras públicas, enseñan educación en emprendimiento, para emprender y desarrollar empresas, 
como fomentan la cultura emprendedora, que estrategias utilizan, con que infraestructura y al hacerlo, entender si aportan o no al crecimiento económico, al desarrollo social y en especial a la superación de la pobreza en Colombia.

En los establecimientos educativos colombianos, incluyendo los de Básica, media y superior, el emprendimiento, desde un enfoque de desarrollo humano integral, le debe permitir a la comunidad educativa e institucional:

- Construir conocimientos y desarrollar hábitos, actitudes y valores necesarios para generar acciones orientadas al mejoramiento personal y a la transformación del entorno y de la sociedad. Las universidades en Colombia con vocación emprendedora e innovadora, cuentan con currículos flexibles, cátedras de emprendimiento, orientadas al desarrollo y fortalecimiento de actitudes y aptitudes emprendedoras, fomentan la investigación formativa para conocer su entorno, han definido el perfil de un emprendedor, el cual cohesionan con la teoría del espíritu emprendedor, de tal manera que orientan la catedra al desarrollo de capacidades creativas, inventivas e innovadoras.

- Dar soluciones a las necesidades humanas presentes en la comunidad, con un sentido de ética y responsabilidad social y una perspectiva de desarrollo sostenible. $\mathrm{Al}$ respecto las instituciones de educación superior que tienen una tradición y alto compromiso con el fomento de la cultura emprendedora y la empresarialidad, cuentan con una conexión entre la investigación, el emprendimiento y la innovación, a partir del fomento de la investigación aplicada, la cual cumple una función social, por tanto identifican problemas, los conocen y promueven nuevo conocimiento para corregir las imperfecciones del sistema que se identifican, es en este sentido que se convierten en universidades socialmente responsables.

- Promover la cooperación y el trabajo en equipo en todos los miembros de la comunidad. El fomento de la cultura emprendedora y la empresarialidad en las universidades seleccionadas, implica el desarrollo articulado y alienado entre la gestión académica, gestión de la comunidad, gestión directiva y la administrativa y financiera. Por tanto, se fomentan capacidades emprendedoras en los ámbitos de los establecimientos educativos, incluyendo las universidades si se determina el contexto social y productivo de la institución, los actores que lo dinamizan y se busca la forma de asignar roles, alienar los comportamientos con un direccionamiento estratégico emprendedor e innovador.

- Fortalecer en los estudiantes la capacidad de conseguir y conservar un empleo, acceder a diferentes alternativas laborales y a posibilidades de autoempleo. La formación integral que prometen las universidades colombianas y los establecimientos educativos en general, premian la autonomía, la superación personal, lo que significa que existe un evidente compromiso con convertir las potencialidades en capacidades para crear empresa o mejorar la efectividad productiva de una existente, por tanto, el autoempleo, la generación de trabajo para otros o la conservación del que se tiene, se logra con facilidad.

- Consolidar procesos de articulación del establecimiento educativo con el sector productivo, la educación superior y la educación para el trabajo. Las universidades 
en Colombia integran mesas de trabajo, están vinculadas a los clúster de transformación productiva, diseñan y desarrollan programas pertinentes, promueven centros de investigación, observatorios, laboratorios dé creatividad e invención, centros de desarrollo empresarial y de la innovación (Al respecto, es importante destacar lo referente a la Red Nacional para el Emprendimiento, la cual existe, y está adscrita al Ministerio de Comercio Industria y Turismo, las universidades seleccionadas, se encuentran afiliadas a esta Red Nacional para el Emprendimiento, por tanto, vienen desarrollando funciones establecidas en la ley).

\section{Conclusiones}

En el marco de la investigación en desarrollo se analizaron 7 universidades privadas y 5 públicas, que en Colombia son reconocidas por poseer lineamientos estratégicos donde declaran su compromiso con el fomento de la cultura emprendedora, el desarrollo del espíritu empresarial y la empresarialidad de los miembros de su comunidad académica. Al analizar el programa de Administración de Empresas, en modalidad presencial, como muestra, se encontró que tienen duración promedio de 9.2 semestres. Todos están organizados por créditos académicos y en promedio cuentan con 155.64, para toda la carrera, organizados y distribuidos curricularmente en una media por programa de 56.3 espacios académicos. En cada una de estas IES y en los programas analizados se contempla la cátedra de emprendimiento como transversal, y a través de ésta, se fomentan el tipo de competencias señaladas en la ley 1014 de 2006. 14.5 es el promedio de créditos académicos dedicados de manera directa a la educación en emprendimiento y corresponden a una media de 4.5 asignaturas. Adicionalmente existen otros espacios académicos dedicados al fomento de la cultura emprendedora y el desarrollo de actitudes y competencias asociadas, que ascienden a un promedio de 33.5 créditos, distribuidos en un promedio de 10.5 espacios. Al respecto se concluye la universidad privada dedica más créditos académicos y espacios a la enseñanza del emprendimiento que la universidad pública.

En este sentido se analizaron los lineamientos estratégicos establecidos para cada programa, encontrando una razón de ser y promesa de valor, que indica un compromiso con formar profesionales integrales, emprendedores e innovadores en un $100 \%$, capaces de generar empresa (lo declara el 100\% de las universidades privadas, pero solo el $60 \%$ de las públicas). Las competencias que se tienen como objetivo desarrollar, a nivel curricular, son: capacidad para planear, organizar, dirigir y controlar, investigar, crear, inventar, innovar, gestión humana, administrativa, financiera, contable, formulación, ejecución y evaluación de proyectos, visión global, liderazgo, gerenciamiento estratégico y prospectivo, aplicación de tecnologías, espíritu emprendedor, capacidad para crear empresas y forjar negocios en escenarios de incertidumbre $y$ en ambientes diversos - dinámicos. Los currículos en general se orienta por los principios de integralidad, transversalidad, flexibilidad, pertinencia, significatividad, innovación y emprendimiento, estando el $25 \%$ definidos en ciclos propedéuticos o terminales pero articulados entre ellos, con contenidos y Syllabus a nivel de espacios de educación en emprendimiento, orientados al desarrollo de actitudes, tales como: visión prospectiva, flexible, audaz, analítico, crítico, innovador, creativo, emprendedor, participativo, Comportamiento autorregulado, asunción al riesgo, autonomía, responsabilidad, orientación al logro, capacidad para identificar oportunidades y recursos, entre otros.

Se puede realizar una conclusión utilizando los aportes de (Kirby, 2004), indicando que los syllabus 
de educación en emprendimiento que se desarrollan en los programas en estudio, educan acerca del emprendimiento (55.5\% del total) y otros para el emprendimiento (44.5\%). Así mismo, siguiendo los postulados de Bechard y Toulouse en (1998), se establece que en el 50.5.\% de los programas se enseña, familiariza y se fomenta una actitud positiva al estudiante, frente al emprendimiento, primordialmente, este tipo de educación se conoce como "sensibilizadora del espíritu emprendedor", un $35.5 \%$, orienta la educación en emprendimiento a la creación de empresas, mientras que un $14 \%$ concentra sus esfuerzos en promover la habilidad para desarrollar empresas pequeñas y en especial garantizar la supervivencia y el crecimiento de estas. Sin embargo, es importante indicar que lo anterior ocurre desde el enfoque de la educación formal universitaria impartida, pero en estas instituciones también se oferta educación no formal, continua y permanente, la cual fomenta la creación de empresas en un $20 \%$, el crecimiento, el desarrollo y la sostenibilidad de pequeñas empresas en un $75 \%$, mientras que un $5 \%$ se caracteriza en formar formadores que capaciten, formen y entrenen a educadores del emprendimiento. En términos generales predomina la enseñanza del emprendimiento externo, en las tipologías sociales, económicas, tecnológicas y con enfoque hacia la sostenibilidad ambiental.

En cuanto a ¿Cómo lo enseñan?, es importante precisar que existen diferencias entre las competencias que se pretenden desarrollar, pero las podemos resumir, utilizando las señaladas por la Universidad de Cartagena y La Tecnológica de Bolívar: competencias emocionales, de liderazgo, logro, técnicas, cognitivas, comunicativas, contextual y valorativas, para crear, innovar y hacerlo a partir de la autonomía, la confianza, la tenacidad, la perseverancia y la responsabilidad. Para tal fin las IES y los programas en estudio, utilizan diferentes modelos y estrategias pedagógicas. $\mathrm{Al}$ respecto resaltamos el caso de la Universidad Tecnológica, quien posee un modelo propio para promover la cultura emprendedora, la creación de empresas y el crecimiento y desarrollo de estas. El modelo que implementan articula diferentes áreas estratégicas de la institución, como son la dirección de investigación e innovación, la vicerrectoría académica y el área de extensión, dinamizando tres etapas: "Generación ideas-aptitud empresarialEXPOIDEAS", el "Plan de Negocios-Capacidad de Riesgo" y la tercera etapa denominada "Fuentes de Capital-Creación de Empresas". Lo referente a las estrategias pedagógicas para la enseñanza del emprendimiento, se identificaron: conferencias, discusiones en grupos y guiadas, la Promoción de ideas (Brainstorming), estudio de casos, seminarios, simposios, foros, incidentes críticos, laboratorios, talleres, Phillips 66, panel, resolución de conflictos, simulacros, proyectos, lúdica, películas, videos, asistencia a eventos científicos, poster, debates, entrevistas, juego gerencial o empresarial, entre otros. Haciendo uso de aulas tradicionales, laboratorios de creatividad e inventiva, de innovación o simplemente de emprendimiento.

Es oportuno destacar los modelos diseñados y utilizados en universidades como los Andes y EAFIT. Los Andes ha creado una fórmula para fomentar el emprendimiento, centrada en el individuo, pero con estrategias que le permitan desarrollar capacidades y actitudes, en articulación con el entorno y de la mano de un equipo, con el cual se construye el modelo de negocios, pero la viabilidad del mismo dependerá de la gestión de recursos y del alto impacto potencial de los emprendimientos. Por su parte EAFIT, ha diseñado la cadena de valor para el desarrollo emprendedor y de la empresarialidad, centrada en una transversalidad de la educación en emprendimiento y una articulación con todo el portafolio de servicios, sumados a esfuerzos extra curriculares, como ferias, concursos, talleres, escuelas de verano, entre otros. 
De la misma forma se determinó que todos los programas analizados definieron la creación de empresas como una opción de grado, así mismo las instituciones se encuentran integradas en la red nacional para el emprendimiento y en términos generales han logrado realizar desde la educación en emprendimiento impartida, importantes aportes a la creación de empresas, la generación de empleo, el crecimiento económico y social de las regiones de Colombia. Al respecto encontramos que la gestión académica y administrativa desarrollada por la UTB, le han permitido que sus egresados en un 15.8\% (Arraut Camargo, Sánchez Drago, \& Novoa Martínez, 2011) tengan como primera actividad laboral, ser empresario, independiente o autónomo, empresa familiar o empleado empresario. El 20.8\% de sus egresados han creado empresa y de estos el $84.2 \%$ se considera el gestor de la idea de negocio. La Universidad EAN cuenta con más de 27 mil egresados, de los cuales el 33 por ciento son empresarios, de acuerdo con el estudio de imagen y egresados, realizado en el año 2012 y 2015 por el Centro Nacional de Consultoría. A nivel de ejemplo la EAN, destaca a Eduardo Ramírez es un joven empresario, egresado del programa de Administración de Empresas en el año 2002. Trabajó en una empresa de vigilancia durante 4 años, y en el 2009 fundó la compañía de seguridad Hoston Colombian Protection que hoy supera los 200 empleados.

En la universidad ICESI, Los egresados de Administración de empresas, son líderes innovadores con perspectiva global, éticos y socialmente responsables, con una sólida formación cuantitativa que participan en la sociedad de manera activa, reflexiva y crítica, mediante la creación de nuevas empresas y el desarrollo de las existentes. Entre otras, se destacan en la Creación, promoción, organización y coordinación de empresas nuevas; gestión de empresas de familia; consultoría independiente, en temas administrativos. Estudios del
CDEE evidencian que cerca del $66 \%$ de los egresados registran actividades de emprendimiento, siendo este el promedio que también caracteriza a las universidades analizadas. En todas las instituciones analizadas, se evidencia la necesidad de acompañar a los emprendedores y lo realizan de la mano de mentores, así mismo declaran muy importante los recursos y capitales semillas para apalancar la empresarialidad, por ejemplo el CESA a finales del 2015, declaro haber acompañado 193 proyectos en pregrado, con el apoyo de 103 mentores de todas las edades, distintas competencias y muy variada experiencia, también 5 proyectos en fase de incubación, que dirige el Centro de Liderazgo de Emprendimiento (CLE) del CESA. Además, por la participación de los egresados que han sido mentores, el CESA alimenta un fondo de financiación al emprendimiento, abierto para estudiantes y egresados, que al culminar con éxito su incubación, y demostrar que pueden producir un alto impacto, reciben un capital semilla de ese fondo.

Otros datos importantes relacionados al respecto de la educación en emprendimiento, la empresarialidad y el desarrollo socioeconómico, son los estudios del GEN Colombia 2014, quien indico que La proporción de colombianos que deciden iniciar una actividad emprendedora y que se mantienen en ella en un lapso de entre 3 y 42 meses, muestra una tendencia creciente al pasar del $21 \%$ en 2010 y 2011 a $23.7 \%$ en 2013 (Este es uno de los hallazgos del estudio general de emprendimiento GEM en sus siglas en inglés, presentado 2 de julio de 2014, por el equipo de Colombia, conformado por la Pontificia Universidad Javeriana Cali, la universidad del Norte de Barranquilla, la Universidad Icesi de Cali y la Universidad de los Andes de Bogotá), lo que se puede complementar con el informe de iNNpulsa y la Universidad Nacional sede Medellín 2017(El estudio se realizó en las capitales de los 32 departamentos y en 10 
municipios de áreas metropolitanas de Medellín, Cali y Bucaramanga), quienes revelaron que en el país hay 2.696 startups que se encuentran en su primera etapa de desarrollo, pero que tienen un alto potencial de éxito. Estos emprendimientos con menos de cinco años de operaciones han generado 7.933 empleos directos (Medellin, s.f.). En el estudio se comprobó que la mayor proporción de los emprendedores está entre los 25 y 35 años (49,37\% de los consultados), seguidos de los que se ubican en el rango de edad entre 35 y 45 años $(28,79 \%)$ de la muestra. Además, el estudio encontró que los hombres $(69,4 \%)$ tienen una mayor participación en el entorno emprendedor. Adicionalmente, el 84,4\% de los colombianos dijo que hoy cuentan con un emprendimiento afirmaron tener un nivel de formación universitaria y el $76,3 \%$ dijo manejar un segundo idioma, en su mayoría el inglés. Los resultados obtenidos permiten constatar que el mayor porcentaje de emprendedores innovan en servicios y productos (53,35\% y $24,4 \%$ respectivamente). De los emprendedores que participaron en el estudio, el $45,44 \%$ señalaron haber formalizado su compañía en un tiempo no mayor a un año, mientras que solo el 6,57\% de los encuestados no ha formalizado aún su negocio. Se identificó que el 89,13\% de los emprendedores utiliza recursos propios como préstamos personales o inversiones familiares para iniciar sus emprendimientos. Igualmente, se percibe una baja participación en el uso de otros recursos como subsidios de entidades gubernamentales nacionales y regionales, fondos de capital de riesgo y otros $(10,87 \%)$.

En conclusión se puede indicar desde la perspectiva descriptiva y explicativa, que la educación en emprendimiento en Colombia, desarrollada por las Universidades, ha logrado fomentar una cultura en emprendedora y de empresarialidad, que ha significado un despliegue de la creatividad y la inventiva de miles de estudiantes y egresados, logrando introducir innovaciones, que han creado empleo directos e indirectos, contribuido a la disminución de la pobreza monetaria y estimulado el desarrollo profesional y humano, facilitando la calidad de vida, el bienestar y la felicidad de estos y parte de su entorno.

\section{Referencias}

Acs, Z., \& Arenius, P. y. (s.f.). Global entrepreneurship Monitor. (2004). Executive Report. En: Babson College and London Business School.

Arendt, H., Cruz, M., \& Novales, R. G. (1993). La condición humana 3. Barcelona: Paidós.

Arraut Camargo, L. C., Sánchez Drago, H., \& Novoa Martínez, K. (2011). Impacto del Programa Emprendedor en la Universidad Tecnológica de Bolívar mediante un análisis a través de SPSS. Revista EAN, 71, 184-194.

Audretsch, D. (2009). Capital emprendedor y crecimiento económico. Revista de investigaciones Regionales. 38 (8), 949-959.

Avellaneda, P. (2007). Movilidad, Pobreza y Exclusión Social. Universidad Autónoma de Barcelona.

Banco Mundial. (2003). Construir Sociedades de Conocimiento: Nuevos Desafíos para la Educación Terciaria. Washington.

Baptistsay, J., Schumpeter, J., \& Baumol, W. (1993). Formal entrepreneurship theory in economics: Existence and Bounds. Journal of Business Venturing. 8, (3).

Bechard, J., \& Toulouse, J. (1998). Validation of a didactic model for the analysis of training objectives in entrepreneurship. Journal of Business Venturing.

Becker, G. S. (2009). Human Capital: A Theoretical and Empirical Analysis, with Special Referencia to Education. $3^{\text {a }}$ edición. Chicago: University of Chicago Press.

Bonal, X. (2004). Contra la pobreza... ¿desigualdad? La educación como estrategia de superación de la pobreza según el Banco Mundial. Algunos ejem- 
plos de América Latina. En: Gomà, R. y Jordana, J. (eds.) Descentralización y política social en América Latina. Barcelona: Fundación CIDOB.

Bonal, X. (2006). Focalización educativa y lucha contra la pobreza: una discusión acerca de los límites y posibilidades del Programa Bolsa Escola, a Bonal, X. (ed.) (en prensa) Globalización, educación y pobreza en América Latina: ¿hacia una nueva agenda política? Barcelona: Fundación CIDOB.

Bonal, X., Tarabini, A., \& Klickowsky, F. (Enero de 2006). ¿Puede la educación erradicar la pobreza? . Cuadernos de Pedagogía.

Boni Aristizábal, A. (2000). La educación superior desde el enfoque de capacidades. Una propuesta para el debate. Revista electrónica interuniversitaria de formación del profesorado, 13(3).

Cantillon, R. (1755). Ensayo sobre la naturaleza del comercio en general.

Casson, M. (1982). The Entrepreneur: an Economic Theory, Martin Robertson. Oxford.

Castillo, A. (1999). Estado del arte e la Enseñanza del emprendimiento. 8.

Congreso de la República de Colombia. (1991). Constitución Política de la República de Colombia Artículo 67 de Junio 4. Bogotá.

Congreso de la República de Colombia. (1991). Decreto 393 de Febrero 8.

Congreso de la República de Colombia. (1991). Decreto 585 de Febrero 26.

Congreso de la República de Colombia. (1991). Ley 29 de Febrero 27.

Congreso de la República de Colombia. (1992). Ley 30 de Diciembre 28 .

Congreso de la República de Colombia. (1994). Ley 115 .

Congreso de la República de Colombia. (2000). Ley 590 de 10 de Julio .

Congreso de la República de Colombia. (2002). Ley 749 .

Congreso de la República de Colombia. (2006). Ley 1014.
Drucker, P. (1964 ). Managing for Results: Economic Tasks and Risk-Taking Decisions.

Gutiérrez Montoya, G. (2011). Educación emprendedora en la universidad: Educando para el futuro. RETOS. Revista de Ciencias de la Administración y Economía, 1(2).

Habermas, J. (2000). Aclaraciones a la ética del discurso. Madrid: Trotta.

Harkavy, I. (2006). The role of universities in advancing citizenship and social justice in the 21st. century. En Education, Citizenship and Social Justice. En: Education, Citizenship and Social Justice.

Howard H., S. (1983). A perspective on entrepreneurship. Harvard Business School Working.

Ibáñez, M. (2001). Actitudes emprendedoras de los estudiantes universitarios de la CAPV. Tesis doctoral de la Facultad de Ciencias Económicas y Empresariales.. Bilbao: Universidad de Deusto.

Kirby, D. A. (2004). Entrepreneurship education: can business school meet the challenge? Education + Training. 46 (8/9), 510-519.

Kirzner, I. (1979). Perception, Opportunity, and Profit: Studies in the Theory of Entrepreneurship. Chicago IL: The University of Chicago Press.

Kirzner, I. M. (1973). Competition and Entrepreneurship. University of Chicago Press.

Knight, F. (1921). Risk, Uncertainty and Profit. Houghton Miffli. New York.

Knight, F. (2006). Risk, uncertainty and profit. New York: Dover Publications.

Krauss, C. (2008). Las actitudes emprendedoras de los estudiantes de la Universidad Católica del Uruguay. (Tesis Doctoral). Universidad de Deusto. España.

Larrañaga, O. (1997). Educación y superación de la pobreza en América Latina. Un estudio del Proyecto Mitigación de la Pobreza y Desarrollo Social del Programa de las Naciones Unidas para el Desarrollo (PNUD) - ISBN: 9978 40-315 9.

Marinelli, F. (Jul/Aug de 1998). Encouraging Visionary Board Leadership, Nonprofi t World USA. 11. 
McGinnis, M., \& Vcmey, T. P. (1987). Innovation management and intrapreneurship, in Sam advanced Managemell Journal. Summer, 19-23.

Medellin, I. (s.f.). https://www.innpulsacolombia.com/ es/mapeo-ecosistema-startup.

Mises, L. V. (1949). Human Action: A Treatise on Economics (Fourth Revised Edition ed.). San Francisco, CA: Fox \& Wilkes.

Nussbaum, M. C. (1997). Cultivating Humanity. A classical defense of reform in liberal education. Massachusetts: Havard University Press.

Primer Congreso Provincial sobre Emprendedorismo y Desarrollo Local, Pigüe. (2003).

Robinson, P. (1987). Prediction of entrepreneurship based on an attitude consistency model. (Tesis doctoral). University of Brigham Young: Provo, Utah.

Salmi, J. (2003). Construction des sociétés du savoir: nouveaux défis pour l'enseignement supérieur en Breton, G. \& Lambert, M. (dir.). Globalisation et Universités. Unesco: Les Presses de l'Université Laval. Paris-Quebec.

Sánchez, J. C., Caggiano, V., \& Hernández, B. (2011). Competencias emprendedoras en la educación universitaria. International Journal of Developmental and Educational Psychology, 3(1), 19-28.

Say, J.-B. (1803). Tratado de Economía Política, o la simple exposición de cómo se forman, se distribuyen y consisten riqueza. Crapelet. 11. (1 st ed ed.).

Schumpeter, J. A. (1934). The Theory of Economic Development. Cambridge, MA: Harvard University Press.

Sen, A. (2000). Desarrollo como Libertad.
Sen, A. K. (1976). Elección colectiva y bienestar social. Alianza Editorial.

Shumpeter, J. (1950). Capitalism, Socialism and democracy. 3era. edicion. New York: Harper y Brothers.

Stevenson H, H. (2000). Why the entrepreneurship has won! Recuperado de: www.usasbe.org/pdf/cwp2000-stevenson.pdf

Stuart Mil, J. (1803). Traité d'économie politique, Traité déconomie politique ou simple exposition de la manière dont se forment, se distribuent et se composent les richesses (1 Ed ed.). Crapelet.

Tarabini, A. (2008). Educación, Pobreza y Desarrollo, Agendas globales, políticas Nacionales y locales. (Tesis Doctoral). Universidad Autónoma de Barcelona.

Timmons, J. (1994). Babson College. Comunicación personal. 1996. Y Shefsky sostiene sus argumentos en su libro: Entrepreneurs are made, notborn (los emprendedores se hacen, no nacen). Mac. Graw Hill .

Torres, B. E. (2009). Capital humano e intelectual: su evaluación. Observatorio Laboral Revista Venezolana. 2 (3), 65-81.

Varela, R., \& Bedoya, O. (2006). Modelo conceptual de desarrollo empresarial basado en competencias. 22(100), 21-48. Recuperado de: Estudios Gerenciales. http://www.icesi.edu.co/revistas/index. php/estudios_gerenciales/article/view/198

Walker, M. (2006). Higher education Pedagogies. Berkshire: The society for research into Higher Education and open University press. 